\title{
Estimation of the anisotropy parameters from imaging moveout of diving wave in a factorized anisotropic medium
}

\author{
Shibo $\mathrm{Xu}^{1}$, Alexey Stovas ${ }^{1}$, and Tariq Alkhalifah ${ }^{2}$
}

\begin{abstract}
The importance of diving waves is being realized because they provide long-wavelength model information, which can be used to help invert for the reflection information in fullwaveform inversion. The factorized model is defined here as a combination of vertical heterogeneity and constant anisotropy, and it admits closed-form description of the traveltime. We have used these resulting analytical formulas to describe the behavior of diving waves in a factorized anisotropic medium, and we used an approximate imaging moveout formulation (residual moveout after imaging) to update the velocity model when the wrong model parameters (isotropic assumption) were used for imaging. We then used these analytical representations of the image moveout to establish a semblance analysis framework to search for the optimal anisotropic parameters. We have also discussed different parameterizations of the factorized medium to find the one that gave the best accuracy in anisotropy parameters estimation.
\end{abstract}

\section{INTRODUCTION}

Recently, direct arrivals and specifically diving waves have been experiencing a new lease on life because they can work as one of the major sources of information for the long-wavelength component of the velocity model (Virieux and Operto, 2009). These inverted models, which are based on diving waves, can provide an initial velocity model that is sufficiently close to the true model within the fullwaveform inversion (FWI) requirements. In a general FWI, we update the velocity model by using the information from the reflected and diving waves. Recently, many have suggested that we focus initially on the diving (transmission) waves and try to isolate them (Tang et al., 2013) because they provide a long-wavelength update for updating the velocity model (Sirgue and Pratt, 2004). A sensitivity analysis to assess the contribution of refracted, reflected, and diving waves for the reconstructed velocity perturbation is studied by Kazei et al. (2013).

The moveout behavior and the focusing of the imaging process for reflected waves have been studied for years, and we have obtained considerable analytical insights of such behavior. Compared with the reflected waves, the imaging of diving waves has lagged behind, and studies in this matter are rare. With the emergence of FWI, the importance of diving waves is now being realized. In fact, the acquisition of large offsets capable of acquiring diving waves is becoming highly desirable because they penetrate at large depths. One of the earliest analyses of the diving waves' acquisition and traveltime can be found in Levin (1996). An approach to measure the defocusing in imaging of diving waves in the subsurface due to velocity errors is analyzed by Shen (2013). In this paper, the update kernel is essentially similar to that experienced for FWI of diving waves. Therefore, this defocusing (residual image moveout) can be used to update the velocity model. Stovas and Alkhalifah (2014) propose using analytical approximations of image moveout of diving waves in a constant-gradient isotropic velocity model to gain a better understanding of the role of diving waves and the update of the velocity model from the defocusing in imaging. We extend this approach to factorized anisotropic models.

The factorized model, assuming vertical heterogeneity and constant anisotropy parameters, is useful in seismic data processing and modeling (Alkhalifah, 1995; Sarkar and Tsvankin, 2003). The raytracing problem in factorized models with elliptic anisotropy is discussed extensively by Rogister and Slawinski (2005). The equations for offset, traveltime, and relative geometric spreading for an analytically described factorized model that combines vertical inhomogeneity with a constant transversely isotropic model with vertical symmetry axis (VTI) are derived by Stovas (2010).

\footnotetext{
Manuscript received by the Editor 4 November 2015; revised manuscript received 9 April 2016; published online 10 June 2016.

${ }^{1}$ Norwegian University of Science and Technology, Department of Petroleum Engineering and Applied Geophysics, Trondheim, Norway. E-mail: shibo.xu@ ntnu.no; alexey.stovas@ntnu.no.

${ }^{2}$ King Abdullah University of Science and Technology, Astronomy and Geophysical Research Institute, Thuwal, Saudi Arabia. E-mail: tariq.alkhlifah@kaust. edu.sa.

(C) 2016 Society of Exploration Geophysicists. All rights reserved.
} 
In this paper, we develop such formulas for diving waves in a factorized VTI medium and analyze their behavior. We define the imaging moveout formulation resulting from using the wrong velocity (isotropic assumption). The explicit equations for imaging moveout are obtained by considering various approximations. We test the accuracy of these approximations for different values of velocity gradients. Synthetic seismic data are used to illustrate this approach. We evaluate the anisotropy parameters by semblance analysis of the depth-migrated data. The parameterization is very important for multiparameter FWI (Alkhalifah and Plessix, 2014). Finally, we discuss the estimation results by adopting different parameterizations.

\section{DIVING WAVES IN A FACTORIZED VTI MEDIUM}

The factorized model (Sarkar and Tsvankin, 2003; Stovas, 2010) involves anisotropy and vertical heterogeneity. In our case, the factorized medium is defined as a transversely isotropic model with a vertical symmetry axis (VTI) under the acoustic approximation (Alkhalifah, 1998). The vertical P-wave velocity is linearly changing with depth, whereas the anisotropy parameters (Thomsen, 1986) remain constant. The vertical velocity in this factorized model is given by

$$
V_{z}=V_{0}+G z
$$

where $V_{0}$ is the P-wave vertical velocity on the surface and $G$ is the gradient of velocity.

For this model, we focus on the diving wave propagation. The fact that the diving wave propagates mostly horizontally points us to a specific choice of parameters to represent the VTI medium. Specifically, we would like to preserve the horizontal wave propagation properties rather than vertical. Therefore, we parameterize the VTI part of the model with the parameters $V_{0}, \varepsilon$, and $\eta$. In the following, we analyze other parameterizations and their effect on the anisotropy parameters estimation.

Based on the parameterization introduced above, the VTI slowness surface can be given by

$$
q(p)=\frac{1}{V_{0}} \sqrt{\frac{1-(1+2 \varepsilon) p^{2} V_{z}^{2}}{1-\frac{2 \eta}{1+2 \eta}(1+2 \varepsilon) p^{2} V_{z}^{2}}}
$$

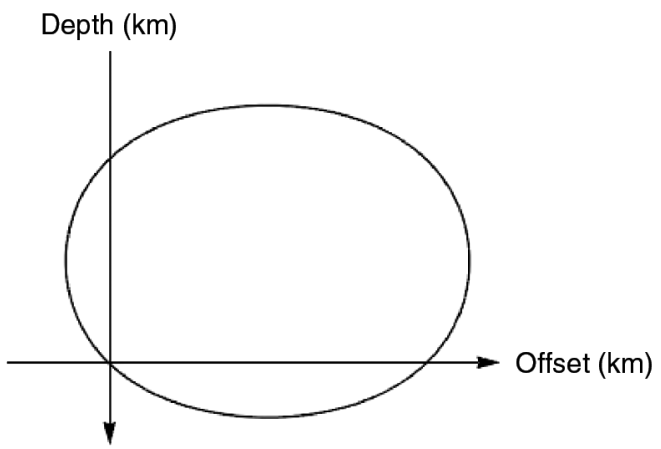

Figure 1. Ray trajectory of diving wave in a factorized VTI medium. where $q$ and $p$ are the vertical and horizontal slownesses, respectively.

Because of the anisotropy, the ray trajectory of the diving wave in a factorized VTI medium is different from the isotropic case and is shown in Figure 1. The shape of the ray is given by an arc of quasiellipse. The ray trajectories are affected by $V_{0}, G$, and the anisotropy parameters. The changes in ray trajectory due to the changes in $V_{0}$,
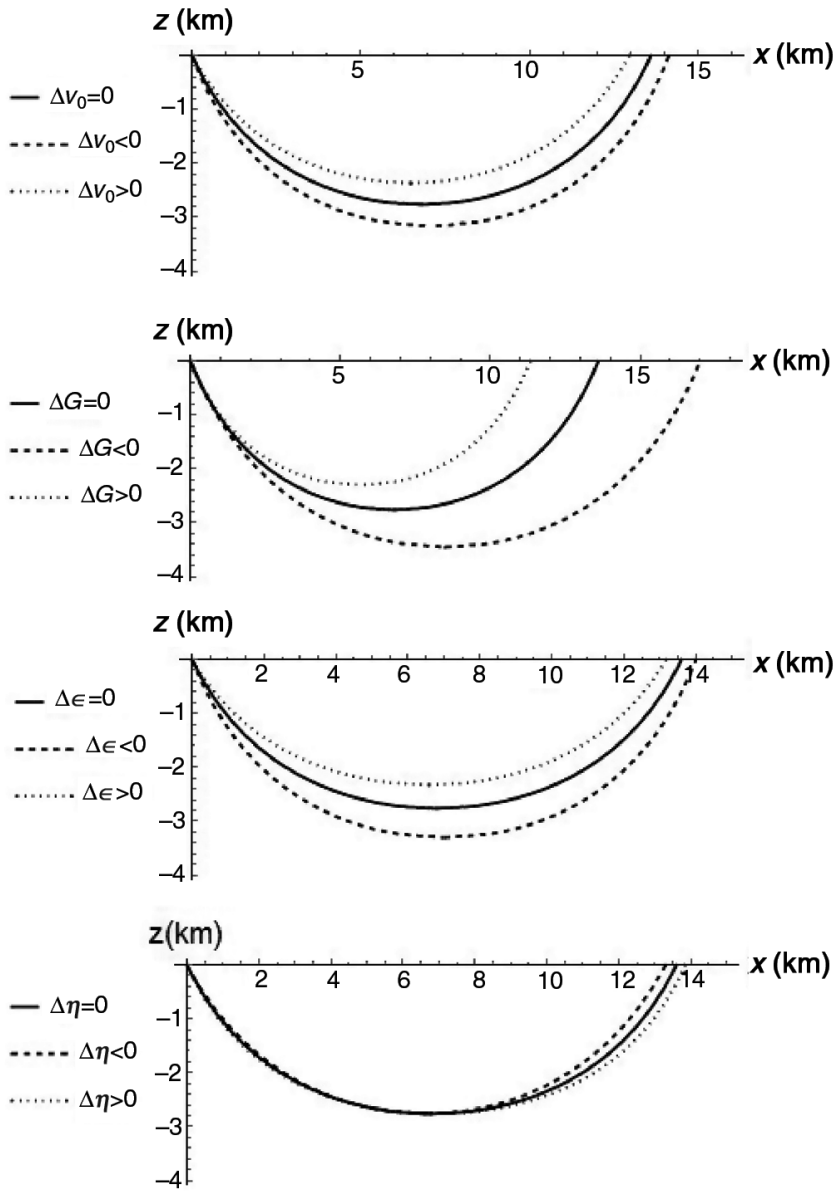

Figure 2. Change in ray trajectory due to perturbations in $V_{0}, G, \varepsilon$, and $\eta$, respectively. The model parameters are $V_{0}=2 \mathrm{~km} / \mathrm{s}$, $G=0.5 \mathrm{~s}^{-1}, \quad \varepsilon=0.2$, and $\eta=0.2$ with perturbations of $\Delta V_{0}= \pm 0.2 \mathrm{~km} / \mathrm{s}, \Delta G=0.1 \mathrm{~s}^{-1}, \Delta \varepsilon= \pm 0.1$ and $\Delta \eta= \pm 0.1$.

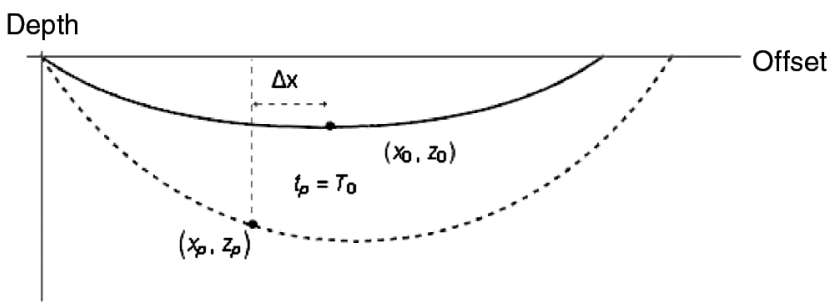

Figure 3. Diving wave imaging moveout (imaging point shift from the turning point). The ray trajectories in factorized VTI and isotropic media are shown by solid and dashed lines, respectively. 
$G, \varepsilon$, and $\eta$ are shown in Figure 2. The model parameters are $V_{0}=2 \mathrm{~km} / \mathrm{s}, G=0.5 \mathrm{~s}^{-1}, \varepsilon=0.2$, and $\eta=0.2$ with considered perturbations: $\Delta V_{0}= \pm 0.2 \mathrm{~km} / \mathrm{s}, \Delta G= \pm 0.1 \mathrm{~s}^{-1}, \Delta \varepsilon= \pm 0.1$, and $\Delta \eta= \pm 0.1$. Compared with the change in the anisotropy parameter $\eta$, the influence of a change in the anisotropy $\varepsilon$ is much more pronounced on the ray geometry.

The position of the turning point $\left(x_{0}, z_{0}\right)$ (Figure 3 ) in a factorized VTI medium can be obtained by setting the vertical slowness to zero, and defined as

$$
x_{0}(p)=\frac{1}{G p} \sqrt{\frac{R_{1}}{R_{2}}}, \quad z_{0}(p)=\frac{1-\sqrt{1+2 \varepsilon} p V_{0}}{\sqrt{1+2 \varepsilon} G p}
$$

where

$$
R_{1}=1-(1+2 \varepsilon) p^{2} V_{0}^{2}, \quad R_{2}=1-\frac{2 \eta}{1+2 \eta}(1+2 \varepsilon) p^{2} V_{0}^{2}
$$

The traveltime from the source position $(0,0)$ to the turning point $\left(x_{0}, z_{0}\right)$ takes the form (Stovas, 2010)

$$
T_{0}(p)=\frac{1}{G}\left(A_{1}+A_{2}+A_{3}\right)
$$

where $A_{1}$ and $A_{2}$ are given by

$$
A_{1}=\sqrt{\frac{R_{1}}{R_{2}}}, \quad A_{2}=\ln \left(\sqrt{1+2 \eta} \frac{\sqrt{R_{1}}+\sqrt{R_{2}}}{\sqrt{1+2 \varepsilon} p V_{0}}\right),
$$

and the expression for $A_{3}$ depends on the sign of $\eta$

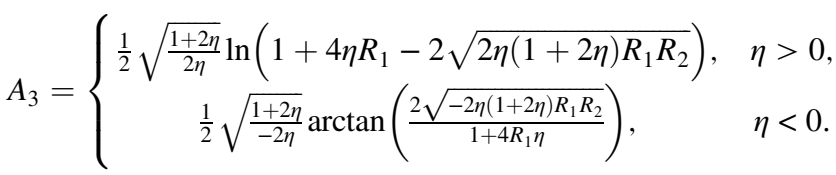

\section{DIVING WAVE IMAGING MOVEOUT}

The definition of diving wave imaging moveout is illustrated in Figure 3. The imaging point of the diving wave, when using the accurate velocity model, will be focused in the turning point position with the coordinates given in equation 3 after applying the imaging condition (Shen, 2013). If the parameters in the velocity model are not accurate, this point will shift and is given by a different ray trajectory defined by the same traveltime and horizontal slowness. This image point dispersal (residual image moveout) can be used to extract information to update the velocity model.

The behavior of diving waves for a constant-gradient velocity model in an isotropic medium has been described analytically by Stovas and Alkhalifah (2014). The traveltime from source $(0,0)$ to a point $\left(x_{p}, z_{p}\right)$ is defined by the relation

$$
t_{p}=\frac{1}{G} \log \left(\frac{V_{0}+G z_{p}}{V_{0}} \frac{1+\sqrt{1-p^{2} V_{0}^{2}}}{1+\sqrt{1-p^{2}\left(V_{0}+G z_{p}\right)^{2}}}\right),
$$

where $z_{p}$ is the corresponding depth of the focusing point with wrong velocity model (isotropic assumption).

We can get the expression of the depth $z_{p}$ by solving the equation $t_{p}=T_{0}$ from equations 5 and 8

$$
\begin{aligned}
& \mathrm{z}_{p}=\frac{V_{0}\left(-b_{0}+b_{1} e^{G T_{0}}+b_{2} e^{2 G T_{0}}+b_{3} e^{3 G T_{0}}+b_{4} e^{4 G T_{0}}\right)}{G\left(b_{0}-b_{2} e^{2 G T_{0}}-b_{4} e^{4 G T_{0}}\right)}, \\
& b_{0}=p^{2} V_{0}^{2}, \quad b_{1}=2\left(1-\sqrt{1-p^{2} V_{0}^{2}}\right), \\
& b_{2}=2\left(p^{2} V_{0}^{2}-2\right), \quad b_{3}=2\left(1+\sqrt{1-p^{2} V_{0}^{2}}\right), \\
& b_{4}=-p^{2} V_{0}^{2},
\end{aligned}
$$

where $T_{0}$ is defined in equations $5-7$. The relation between $x_{p}$ and $z_{p}$ in an isotropic medium with a linearly increasing velocity is given in Stovas and Alkhalifah (2014):

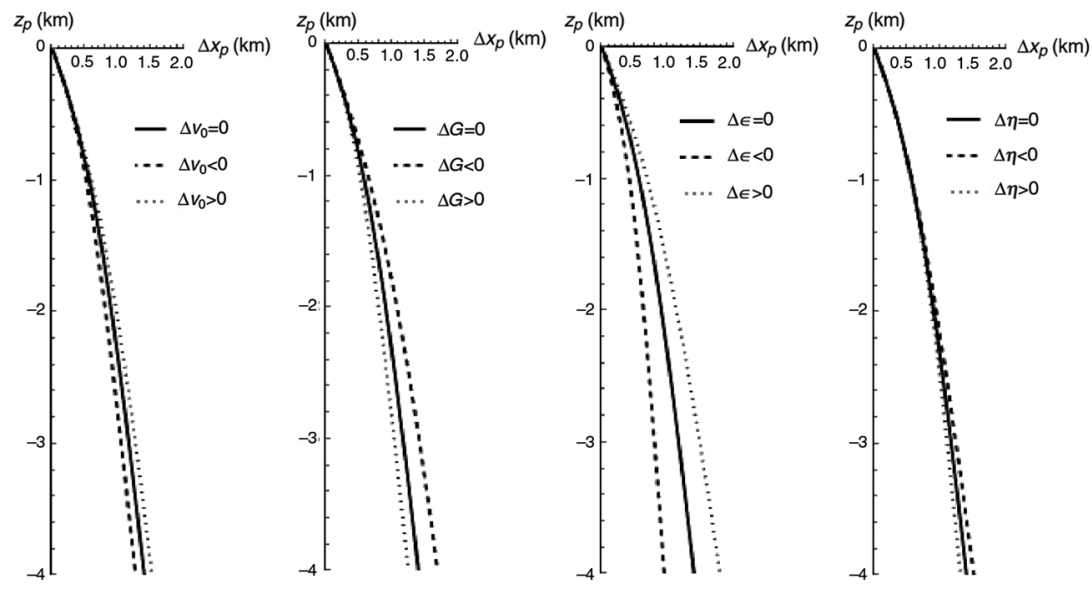

Figure 4. Exact imaging moveout with the perturbations in $V_{0}, G$, and anisotropy parameters $\varepsilon$ and $\eta$. The parameters are $V_{0}=2 \mathrm{~km} / \mathrm{s}, G=0.5 \mathrm{~s}^{-1}$, $\varepsilon=0.3$, and $\eta=0.15$, and with the perturbations are $\Delta V_{0}= \pm 0.5 \mathrm{~km} / \mathrm{s}, \Delta G=0.2 \mathrm{~s}^{-1}, \Delta \varepsilon= \pm 0.1$, and $\Delta \eta= \pm 0.1$. 


$$
x_{p}=\frac{1}{G p}\left(\sqrt{1-p^{2} V_{0}^{2}}-\sqrt{1-p^{2}\left(V_{0}+G z_{p}\right)^{2}}\right) .
$$

We can get a similar approximate expression for VTI media by substituting equation 9 into equation 10 .

The difference in the lateral position of the imaging point, $\Delta x_{p}=x_{0}-x_{p}$, where $x_{0}$ is defined in equations 3 and 4, can be represented as a function of the vertical depth $z_{p}$, which is the so-called diving wave imaging moveout. The functions $\Delta x_{p}$ and $z_{p}$ are the parametric equations represented in terms of the horizontal slowness $p$.

As it is shown in Figure 4, one can see the exact imaging moveout with the perturbations in $V_{0}, G, \varepsilon$, and $\eta$ with the parameters: $V_{0}=2 \mathrm{~km} / \mathrm{s}, G=0.5 \mathrm{~s}^{-1}, \varepsilon=0.3$, and $\eta=0.15$, and the perturbations: $\Delta V_{0}= \pm 0.5 \mathrm{~km} / \mathrm{s}, \quad \Delta G= \pm 0.2 \mathrm{~s}^{-1}, \Delta \varepsilon= \pm 0.1$, and $\Delta \eta= \pm 0.1$. We can see from the plots that $\Delta x_{p}$ increases with vertical velocity $V_{0}$ and anisotropy parameter $\varepsilon$, whereas decreases with gradient $G$ and anisotropy parameter $\eta$. From the plots in Figure 4, we observe that the imaging moveout is very insensitive to the changes in anisotropy parameter $\eta$ under the parameterizations $V_{0}$, $\varepsilon$, and $\eta$.

\section{IMAGING MOVEOUT APPROXIMATIONS}

To choose the best approximation for the imaging moveout, we analyze three types of approximations for $\Delta x_{p}\left(z_{p}\right)$ : the fourth-order Taylor series, the Padé approximation, and the rational approximation.

First, we obtain the analytical Taylor series expansion in depth $z_{p}$, which takes the form (see Appendix A)

a)

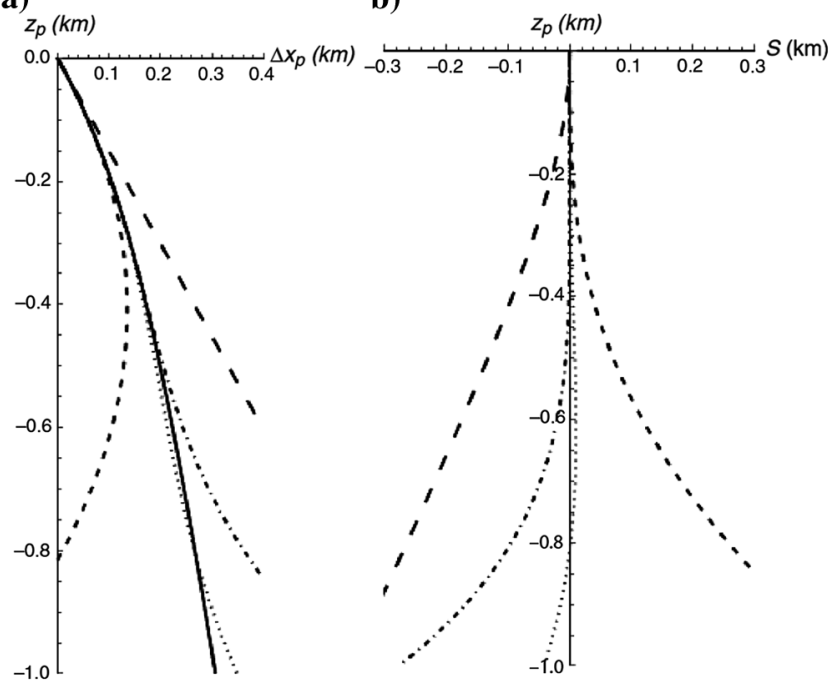

Figure 5. The limited Taylor series approximations from (a) equation 11 and (b) their errors. The one-, two-, three-, and four-term approximations are shown by large dashed, tiny dashed, dotted, and dashed-dotted lines, respectively. The exact imaging moveout curve is shown by solid line. The parameters are $V_{0}=2 \mathrm{~km} / \mathrm{s}$, $G_{1}=1.5 \mathrm{~s}^{-1}, \varepsilon=0.22$, and $\eta=0.1$. The error in imaging moveout is $S=\Delta x_{\text {exact }}\left(z_{p}\right)-\Delta x_{\text {app }}\left(z_{p}\right)$.

a)

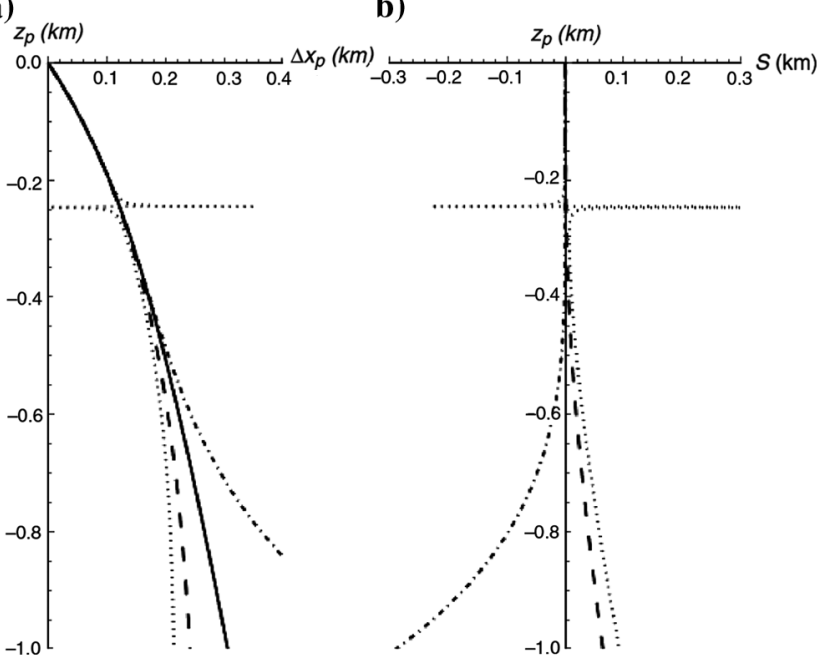

Figure 6. (a) The imaging moveout of three types of Padé approximation $P[2,2], P[2,3], P[3,3]$ and (b) their errors are shown by dashed, dotted, and dashed-dotted lines, respectively. The exact imaging moveout curve is shown by solid line. The parameters are $V_{0}=2 \mathrm{~km} / \mathrm{s}, G_{1}=1.5 \mathrm{~s}^{-1}, \varepsilon=0.22$, and $\eta=0.1$. The error in imaging moveout is $S=\Delta x_{\text {exact }}\left(z_{p}\right)-\Delta x_{\text {app }}\left(z_{p}\right)$.

a)

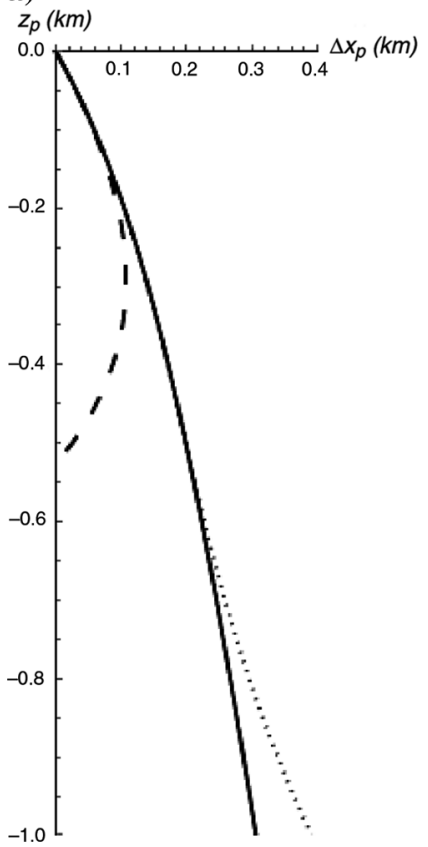

Figure 7. (a) The imaging moveout of two rational approximations in equations 15 and 16 and (b) their errors are shown in dashed and dotted lines, respectively. The exact imaging moveout curve is shown by solid line. The parameters are $V_{0}=2 \mathrm{~km} / \mathrm{s}$, $G_{1}=1.5 \mathrm{~s}^{-1}, \varepsilon=0.22$, and $\eta=0.1$. The error in imaging moveout is $S=\Delta x_{\text {exact }}\left(z_{p}\right)-\Delta x_{\text {app }}\left(z_{p}\right)$. b)

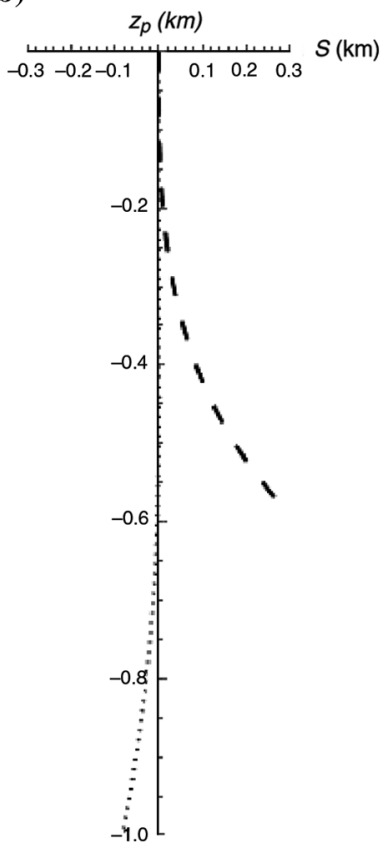




$$
\Delta x_{p}=a_{1} z_{p}+a_{2} z_{p}^{2}+a_{3} z_{p}^{3}+a_{4} z_{p}^{4}
$$

with the series coefficients

$$
\begin{aligned}
& a_{1}=\sqrt{2} \sqrt{\varepsilon}, \quad a_{2}=-\frac{(1+2 \varepsilon) G}{2 \sqrt{2} \sqrt{\varepsilon} V_{0}} \\
& a_{3}=\frac{(1+2 \varepsilon)(-\eta+\varepsilon(3+4 \eta)) G^{2}}{6 \sqrt{2} \varepsilon^{3 / 2} V_{0}^{2}(1+2 \eta)} \\
& a_{4}=\frac{(1+2 \varepsilon)\left(1-2 \eta-16 \varepsilon^{2}(1+\eta)+\varepsilon(2+4 \eta)\right) G^{3}}{32 \sqrt{2} \varepsilon^{5 / 2} V_{0}^{3}(1+2 \eta)} .
\end{aligned}
$$

The first two coefficients in equation 12 do not depend on the anisotropy parameter $\eta$. It confirms our observation on the weak dependency of the imaging moveout on this parameter. To provide an accurate approximation, the Padé approximation is adopted to stabilize the series in equation 11 . We define a Padé approximation $P[i, j]$ to be the rational function given by

$$
P[i, j]=\frac{M_{i}\left(z_{p}\right)}{N_{j}\left(z_{p}\right)}=\frac{m_{1} z_{p}+m_{2} z_{p}^{2}+\cdots m_{i} z_{p}^{i}}{1+n_{1} z_{p}+n_{2} z_{p}^{2}+\cdots n_{j} z_{p}^{j}} .
$$

The series coefficients in the Padé approximation are associated with the Taylor series in equation 11 for $A(z)=\Delta x_{p}=\sum_{j=}$ $1^{4} a_{j} z_{p}^{j}$, and the series coefficients in equation 13 can be calculated by $A(z)-P[i, j]=O\left(z^{i+j}\right)$. If $i+j>4$, the corresponding coefficient of $z^{i+j}$ in the Taylor series will be zero, $a^{i+j}=0$ (Baker et al., 1961).

We analyze three types of Padé approximations, i.e., $P[2,2]$, $P[2,3]$, and $P[3,3]$ for the diving wave imaging moveout $\Delta x_{p}\left(z_{p}\right)$.

To define a more stable and accurate approximation, we compute the infinite depth limit

$$
a_{\infty}=\lim _{z_{p} \rightarrow \infty}\left(\frac{\Delta x_{p}}{z_{p}}\right) \text {. }
$$

Then, we define two types of rational approximation in the following forms:

$$
\begin{gathered}
R_{1}=\frac{p_{1} z_{p}+p_{2} z_{p}^{2}}{1+q_{1} z_{p}}, \\
R_{2}=\frac{P_{1} z_{p}+P_{2} z_{p}^{2}+P_{3} z_{p}^{3}}{1+Q_{1} z_{p}+Q_{2} z_{p}^{2}},
\end{gathered}
$$

where the coefficients $p_{1}, p_{2}, q_{1}, P_{1}, P_{2}, P_{3}, Q_{1}$, and $Q_{2}$ are represented in terms of the Taylor series coefficients in equation 12 and the infinite limit term $a_{\infty}$ (Appendix B).

\section{NUMERICAL EXAMPLES}

To test the accuracy of different approximations, we choose a factorized model with the parameters: $V_{0}=2 \mathrm{~km} / \mathrm{s}, G=1.5 \mathrm{~s}^{-1}$, $\varepsilon=0.22$, and $\eta=0.1$.

First, we test the accuracy of Taylor series approximation with different terms and their errors $S$ in Figure 5. The error function is the difference between exact imaging moveout $\Delta x_{\text {exact }}\left(z_{p}\right)$ and the approximation one $\Delta x_{\text {app }}\left(z_{p}\right)$ with $S=\Delta x_{\text {exact }}\left(z_{p}\right)-$ $\Delta x_{\text {app }}\left(z_{p}\right)$. From the plots in Figure 5, we can see that in the presence of anisotropy, the third-order Taylor series approximation has higher accuracy.

In Figure 6, we show three types of Padé approximation $P[2,2]$, $P[2,3], P[3,3]$, and their error plots. From the plots in Figure 6, one can see that the Padé approximation $P[2,2]$ is more accurate than

a)

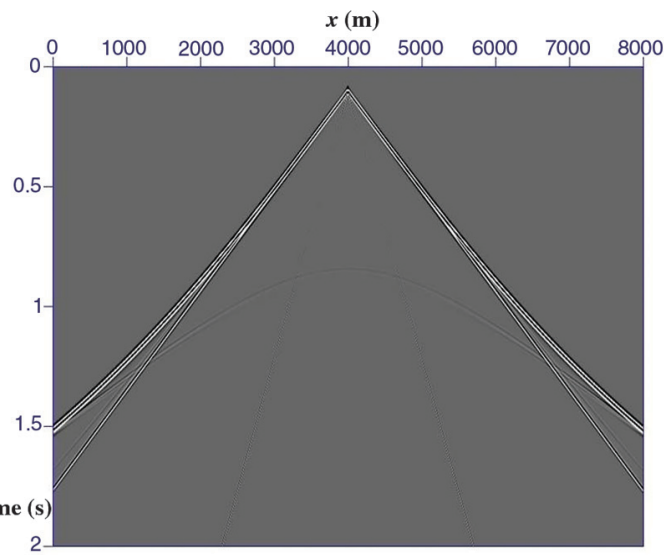

b)
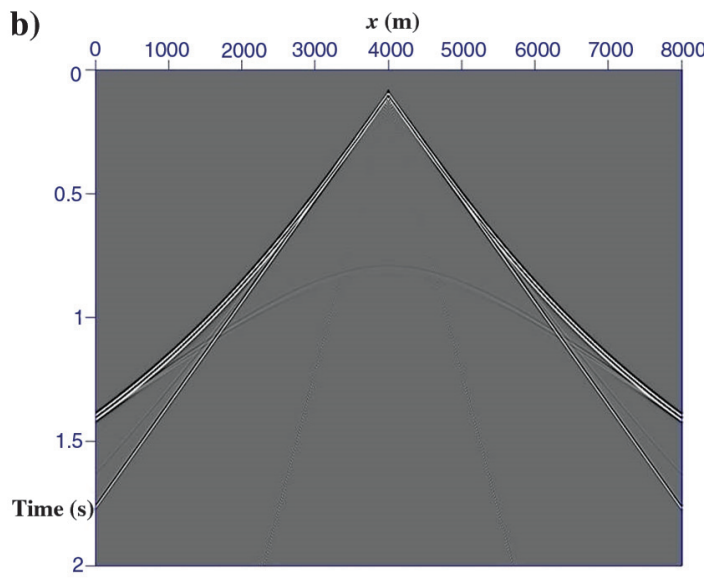

Figure 8. (a) The common-shot gather for the diving wave from the synthetic data with the parameters $V_{0}=2 \mathrm{~km} / \mathrm{s}, G_{1}=1.5 \mathrm{~s}^{-1}$, $\varepsilon=0.22$, and $\eta=0.1$. (b) The common-shot gather for the diving wave from the synthetic data with the parameters $V_{0}=2 \mathrm{~km} / \mathrm{s}$, $G_{2}=2 \mathrm{~s}^{-1}, \varepsilon=0.22$, and $\eta=0.1$. 
the other two, whereas $P[2,3]$ is unstable (Figure 6) because the denominator can vanish at a certain depth.

The imaging moveout of two rational approximations in equations 15 and 16 is shown in Figure 7. From the error plots in Figure 7 , one can see that the rational approximation $R_{2}$ is very accurate. It is almost as accurate as the exact solution up to a depth of $0.6 \mathrm{~km}$. We select the rational approximation $R_{2}$ as our approximation for the upcoming examples.

\section{SEMBLANCE ANALYSIS}

First, we test the accuracy on two common-shot synthetic gathers corresponding to different values of the gradient (Figure 8) $G_{1}=1.5 \mathrm{~s}^{-1}$ (model a) and $G_{2}=2 \mathrm{~s}^{-1}$ (model b). The remaining parameters are $V_{0}=2 \mathrm{~km} / \mathrm{s}, \varepsilon=0.22$, and $\eta=0.1$. The source is located at the surface at lateral position $4 \mathrm{~km}$, with receivers spanning the whole surface. Imaging the shot gather using reverse time migration (RTM) with a space lag imaging condition allows us to obtain the common image gathers. Figure 9 shows the common image gathers from applying the RTM with the accurate parameters. There is no residual imaging moveout, when the exact velocity model is used. In Figure 10, we show the residual imaging moveout when applying RTM under the isotropic assumption on the data shown in Figure 8. For the isotropic model, we keep the same $V_{0}$ and gradients as that of the anisotropic models and set $\varepsilon$ and $\eta$ to zero. The use of wrong anisotropy parameters causes the residual imaging moveout (Figure 10). We overlay the numerically computed imaging moveout with the curves corresponding to our analytical second-order rational approximation $R_{2}$ given in equation 16 . We represent the numerically computed imaging moveout in Figure 10 with a yellow color. Although this equation is approximate, it can accurately predict the residual curve of the imaging

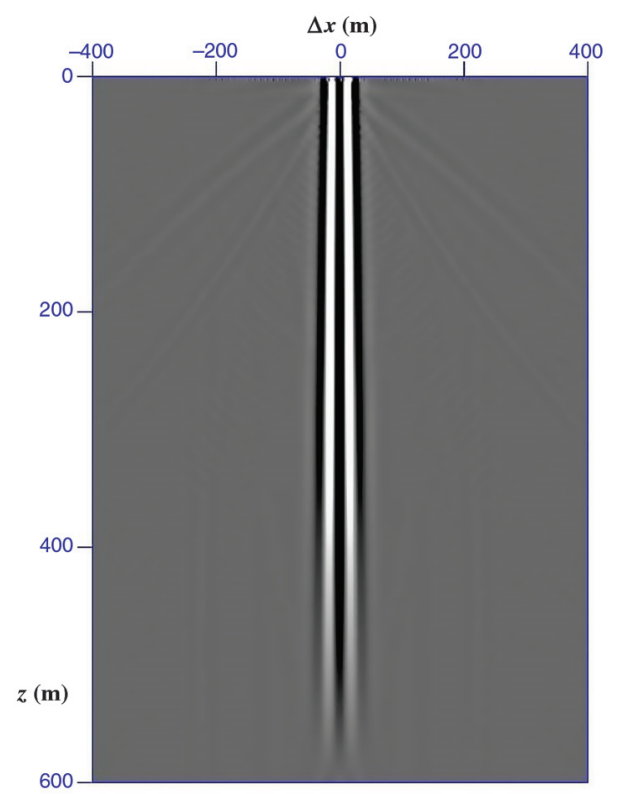

Figure 9. The common image gather for the diving wave when applying the anisotropic RTM with the accurate parameters. moveout for both models. For a larger gradient, the matching between the analytical approximation and RTM is not as accurate with the increasing difference at large depths (Figure 10b). As the typical velocity gradients lie between 0.5 and $1.0 \mathrm{~s}^{-1}$, so our results are acceptable.

We apply the semblance analysis on the RTM result based on the analytical prediction corresponding to the rational approximation $R_{2}$. The equation for the semblance coherency function is given by

$$
\mathrm{SB}=\frac{\sum_{j}^{n z} A_{i(j), j}^{2}}{\left(\sum_{j}^{n z} A_{i(j), j}\right)^{2}},
$$

a)

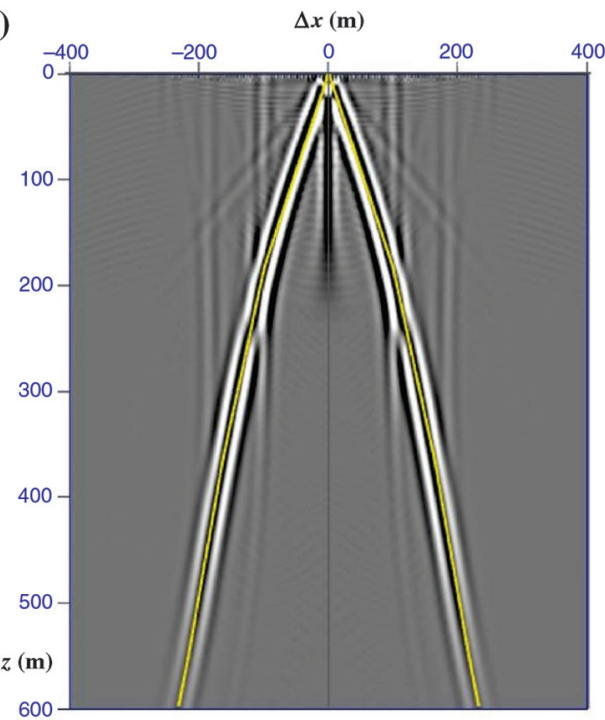

b)

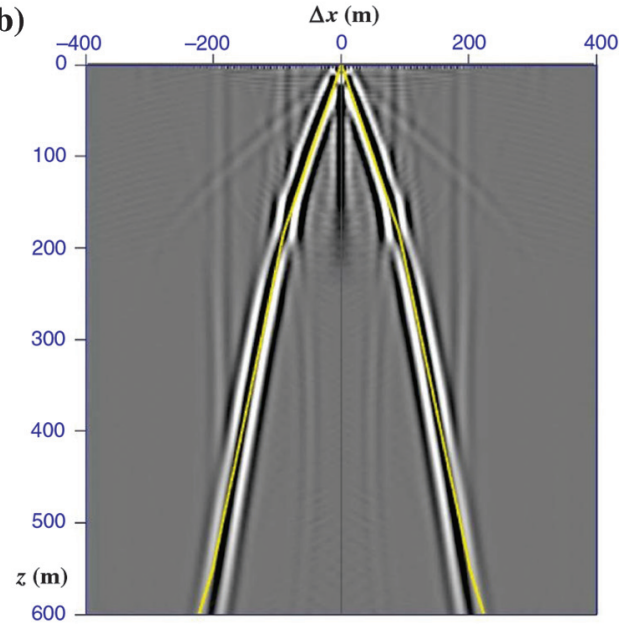

Figure 10. (a) The common image gather when using the isotropic RTM with parameters $V_{0}=2 \mathrm{~km} / \mathrm{s}, G_{1}=1.5 \mathrm{~s}^{-1}, \varepsilon=0$, and $\eta=0$. (b) The common image gather when using the isotropic RTM with parameters $V_{0}=2 \mathrm{~km} / \mathrm{s}, G_{2}=2 \mathrm{~s}^{-1}, \varepsilon=0$, and $\eta=0$. We overlay the residual curve predicted by the imaging moveout from rational approximation $R_{2}$ in equation 16 . 
where SB is the semblance value, $A_{i, j}$ is the amplitude of the RTM data, $i(j)$ is the discretized representation of $x_{p}\left(z_{p}\right)$, and $j$ is the discretized representation of $z_{p}$ in the rational approximation $R_{2}$ given in equation 16. To analyze the influence of VTI medium parameterization, we test a range of anisotropy combinations (see Appendix C) and show the errors in estimation of anisotropy parameters $\Delta \varepsilon$ and $\Delta \eta$ for gradient $G_{1}$ in Table 1 (see Appendix C). From this table, we can see that the estimates from all the examples are reasonably accurate, and the differences between them are very small. Then, we apply the same analysis for the larger gradient $G_{2}$ and show the results in Table 2. The anisotropy estimates for this model are less accurate because the approximation in equation 16 deviates from the RTM result at larger depths as shown in Figure 10b. However, the results are still sufficiently accurate for use as a potential initial model for FWI. Based on the accuracy of estimation of $\tilde{\varepsilon}$ and $\tilde{\eta}$, we select the four best parameterizations: $\left(V_{N}, \varepsilon, \eta\right),\left(V_{N}, \varepsilon, \delta\right), \quad\left(V_{0}, \varepsilon, V_{H}\right)$, and $\left(V_{N}, \varepsilon, V_{H}\right)$ for semblance plots.

In Figure 11, the semblance plots computed for the depth range of 0-600 $\mathrm{m}$ for these four types of parameterizations, where $V_{0}, V_{N}$, and $V_{H}$ are the vertical, NMO, and horizontal velocities, respectively, on the top for gradient $G_{1}$, demonstrate the variations with different parameterizations. The anisotropy parameters can be

Table 1. The anisotropy estimation errors for gradient $G_{1}$ for all parameterizations. We fix the first value of each parameterization and evaluate the other two through semblance analysis, and then we convert them into the estimation for anisotropy parameters $\tilde{\varepsilon}$ and $\tilde{\eta}$.

$$
G_{1}=1.5 \mathrm{~s}^{-1}
$$

One velocity + two anisotropy parameters

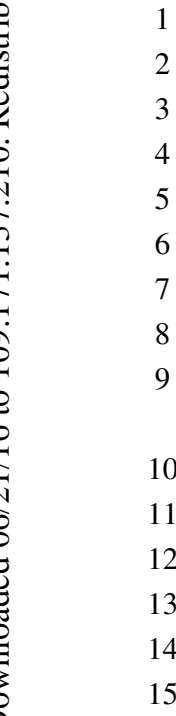

$V_{0}, \varepsilon, \eta$

$\Delta \varepsilon \approx 0.002$

$V_{0}, \delta, \eta$

$\Delta \varepsilon \approx 0.0015$

$V_{0}, \delta, \varepsilon$

$\Delta \varepsilon \approx 0.002$

$V_{N}, \varepsilon, \eta$

$\Delta \varepsilon \approx 0.0025$

$V_{N}, \delta, \eta$

$\Delta \varepsilon \approx 0.002$

$V_{N}, \delta, \varepsilon$

$\Delta \varepsilon \approx 0.0025$

$V_{H}, \varepsilon, \eta$

$\Delta \varepsilon \approx 0.002$

$V_{H}, \delta, \eta$

$\Delta \varepsilon \approx 0.0015$

$V_{H}, \delta, \varepsilon$

$\Delta \varepsilon \approx 0.002$

Two velocities + one anisotropy parameter

$\begin{array}{lc}V_{0}, \varepsilon, V_{N} & \Delta \varepsilon \approx 0.002 \\ V_{0}, \eta, V_{N} & \Delta \varepsilon \approx 0.0016 \\ V_{0}, \delta, V_{H} & \Delta \varepsilon \approx 0.0018 \\ V_{0}, \eta, V_{H} & \Delta \varepsilon \approx 0.0018 \\ V_{N}, \delta, V_{H} & \Delta \varepsilon \approx 0.002 \\ V_{N}, \varepsilon, V_{H} & \Delta \varepsilon \approx 0.0025 \\ V_{H}, \delta, V_{N} & \Delta \varepsilon \approx 0.0016 \\ V_{H}, \varepsilon, V_{N} & \Delta \varepsilon \approx 0.002\end{array}$

Three velocities

$V_{0}, V_{N}, V_{H}$

$\Delta \varepsilon \approx 0.0018$ evaluated from the coordinates by picking the maximal value of the semblance plots.

The semblance plots for a larger gradient $G_{2}$ are shown in Figure 12. Comparing with the semblance plots in Figure 11, we observe that the semblance anomalies are more focused, whereas the estimation results are less accurate. The trade-off between anisotropy parameters also decreases.

\section{DISCUSSION}

Despite the fact that the linear velocity model used for our analysis is an approximation to the real case, it can be used as a part of a factorized model for many real situations. Such as in the moveout approximations for reflections, in which the medium is assumed to be homogeneous to yield such approximations, for diving waves, the vertical increase of velocity is a necessary ingredient for recording such waves. As a result, we develop the analytical approximations based on a constant gradient assumption, which will allow for closed-form solutions. In the same way as Dix-type approximations are used to describe the reflection moveout in a $V(z)$ medium, similar formulas can be derived for the diving wave moveout, where the gradient is varying with depth. Such studies can be a topic of future investigations.

Table 2. The anisotropy estimation errors for gradient $G_{2}$ for all parameterizations. We fix the first value of each parameterization and evaluate the other two through semblance analysis, and then we convert them into the estimation for anisotropy parameters $\tilde{\varepsilon}$ and $\tilde{\eta}$.

$$
G_{2}=2 \mathrm{~s}^{-1}
$$

$\Delta \eta \approx-0.004$

$\Delta \eta \approx-0.0055$

$\Delta \eta \approx-0.0035$

$\Delta \eta \approx-0.005$

$\Delta \eta \approx 0.008$

$\Delta \eta \approx-0.0045$

$\Delta \eta \approx-0.0045$

$\Delta \eta \approx-0.006$

$\Delta \eta \approx-0.0045$
One velocity + two anisotropy parameters

$\begin{array}{llc}V_{0}, \varepsilon, \eta & \Delta \varepsilon \approx 0.0075 & \Delta \eta \approx 0.0385 \\ V_{0}, \delta, \eta & \Delta \varepsilon \approx 0.0080 & \Delta \eta \approx 0.0385 \\ V_{0}, \delta, \varepsilon & \Delta \varepsilon \approx 0.0075 & \Delta \eta \approx 0.045 \\ V_{N}, \varepsilon, \eta & \Delta \varepsilon \approx 0.0045 & \Delta \eta \approx 0.0370 \\ V_{N}, \delta, \eta & \Delta \varepsilon \approx 0.0060 & \Delta \eta \approx 0.0380 \\ V_{N}, \delta, \varepsilon & \Delta \varepsilon \approx 0.0045 & \Delta \eta \approx-0.0355 \\ V_{H}, \varepsilon, \eta & \Delta \varepsilon \approx 0.0080 & \Delta \eta \approx 0.0385 \\ V_{H}, \delta, \eta & \Delta \varepsilon \approx 0.0085 & \Delta \eta \approx 0.0380 \\ V_{H}, \delta, \varepsilon & \Delta \varepsilon \approx 0.0080 & \Delta \eta \approx-0.0215\end{array}$

Two velocities + one anisotropy parameter

$V_{0}, \varepsilon, V_{N} \quad \Delta \varepsilon \approx 0.0075 \quad \Delta \eta \approx 0.0377$

$V_{0}, \eta, V_{N} \quad \Delta \varepsilon \approx 0.0083$

$\Delta \eta \approx 0.0385$

$V_{0}, \delta, V_{H}$

$\Delta \varepsilon \approx 0.0072$

$\Delta \eta \approx 0.0367$

$V_{0}, \eta, V_{H}$

$\Delta \varepsilon \approx 0.0072$

$\Delta \eta \approx 0.0375$

$\Delta \eta \approx-0.005$

$V_{N}, \delta, V_{H}$

$\Delta \varepsilon \approx 0.0060$

$\Delta \eta \approx 0.0380$

$\Delta \eta \approx-0.005$

$V_{N}, \varepsilon, V_{H}$

$\Delta \varepsilon \approx 0.0045$

$\Delta \eta \approx 0.0370$

$\Delta \eta \approx-0.0044$

$V_{H}, \delta, V_{N}$

$\Delta \varepsilon \approx 0.0082855$

$\Delta \eta \approx 0.0377855$

$\Delta \eta \approx-0.0044$

$V_{H}, \varepsilon, V_{N}$

$\Delta \varepsilon \approx 0.0080$

$\Delta \eta \approx 0.0383862$

Three velocities

$\Delta \eta \approx-0.0048$ 
Despite that diving waves admit low-frequency long-wavelength energy over the whole wavepath when imaging, it is stationary near the turning point, so the contributions from many source and receivers at the end allow the turning point part to provide the major contribution and at the high-frequency asymptotic limit, we end up with the turning ray contribution. We definitely do not get energy focused only at the turning point because the classic image for turning waves from a source to a receiver is of a banana shape. However, we are focusing our analysis at the center, which includes the turning point, because it represents the deepest level in which a diving wave travels shown in Figure 13. Using different intervals, we sum all the sources and receivers that covering the surface, the turning point represents the major stationary contribution to the image. The subsurface offset gathers from the left and right parts of the model are shown in Figure 14a and 14b, respectively, using the correct velocity in migration. They need to be compared with Figure 9. We can see that they all focused at the center of corresponding gather. The basic idea is that the increased focusing of image in subsurface off-

a)

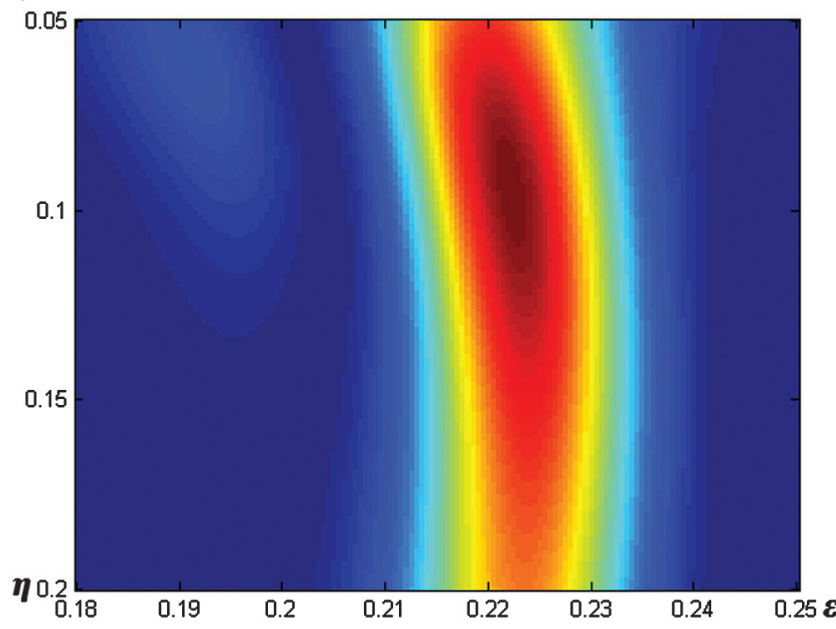

b)

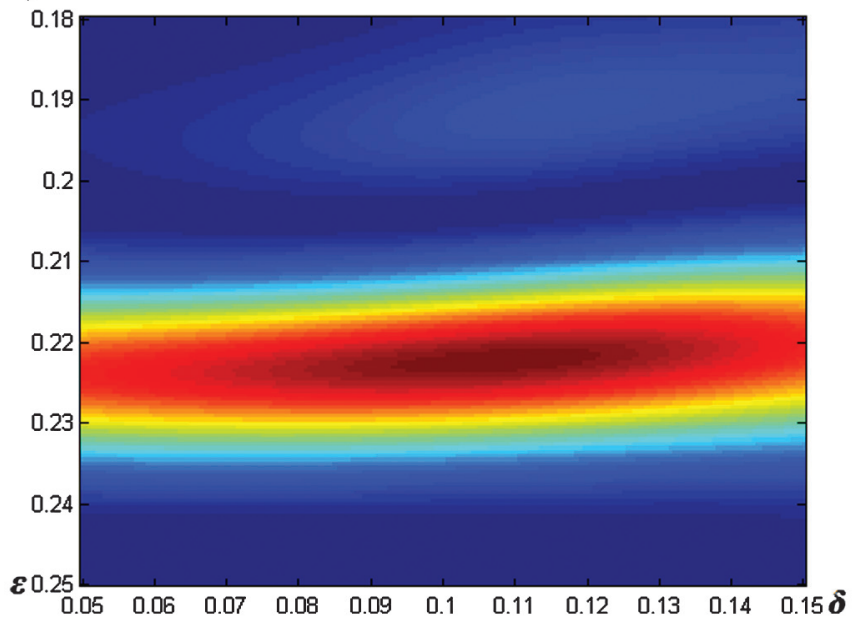

set is induced by the increased degree of similarity between the migration velocity and the true velocity. This is equivalent to what we see in extending the image with an offset lag as we demonstrate in Figures 9 and 10 similar to Shen (2013). Therefore, the image point dispersal is equivalent to the nonfocusing to zero offset we obtain when we look at extended images. In FWI for diving waves, such dispersal depending on the frequency suggests to us the depth at which the gradient can be trusted, and thus, it provides incorrect direction updates when the dispersal exceeds half of the wavelength.

Using the rational approximation, we ended up with relatively small errors under the assumptions made. However, as expected, we observe a trade-off between the anisotropy parameters in the estimation (Figures 11 and 12). The trade-off is an ongoing challenge in analyzing multiparameter problems, such as those in anisotropy. However, with analytical formulas, the computation of the Hessian becomes straightforward, which provides for another opportunity to benefit from these approximations. c)

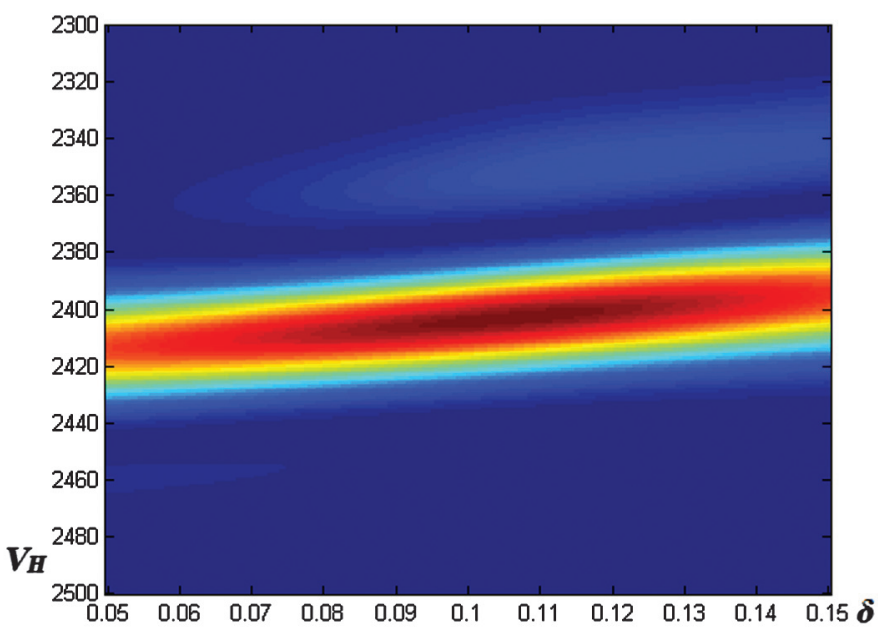

d)

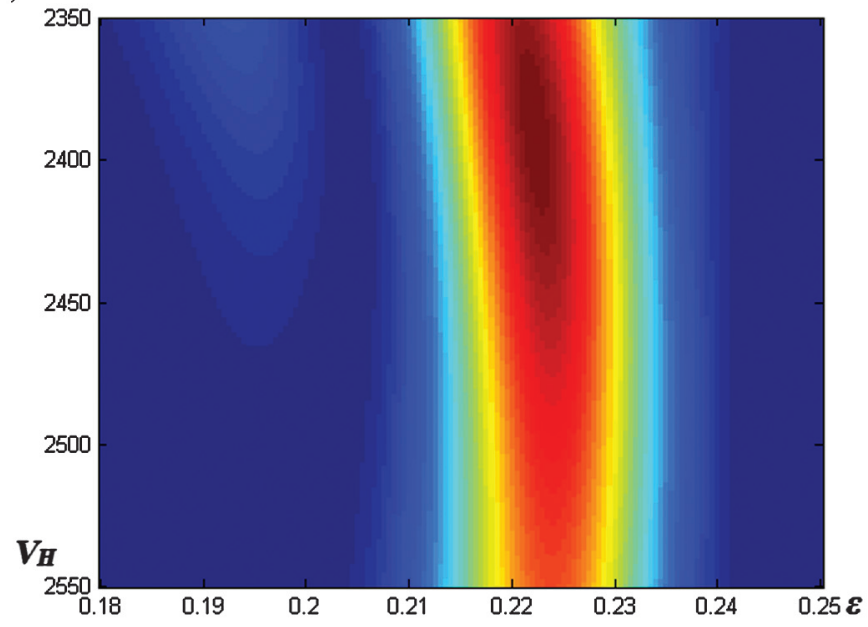

Figure 11. The semblance plots for different parameterizations computed for factorized model with $G_{1}=1.5 \mathrm{~s}^{-1}$ (a) $V_{N^{-}}$fixed, $\varepsilon, \eta$; (b) $V_{N^{-}}$ fixed, $\varepsilon, \delta$; (c) $V_{0}$-fixed, $\delta, V_{H}$; and (d) $V_{N}$-fixed, $\varepsilon, V_{H}$. The anisotropy parameters can be evaluated from the coordinates of the maximal value of the semblance plot. 
a)

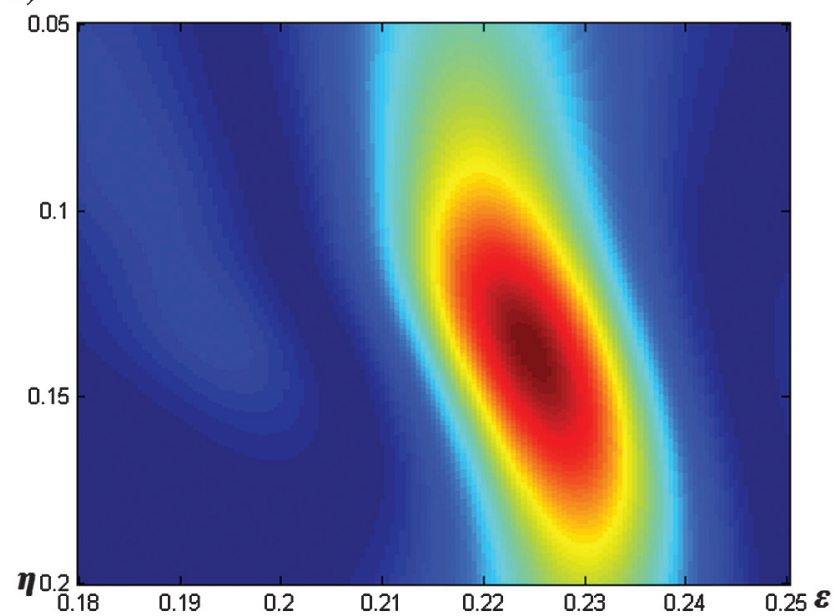

b)

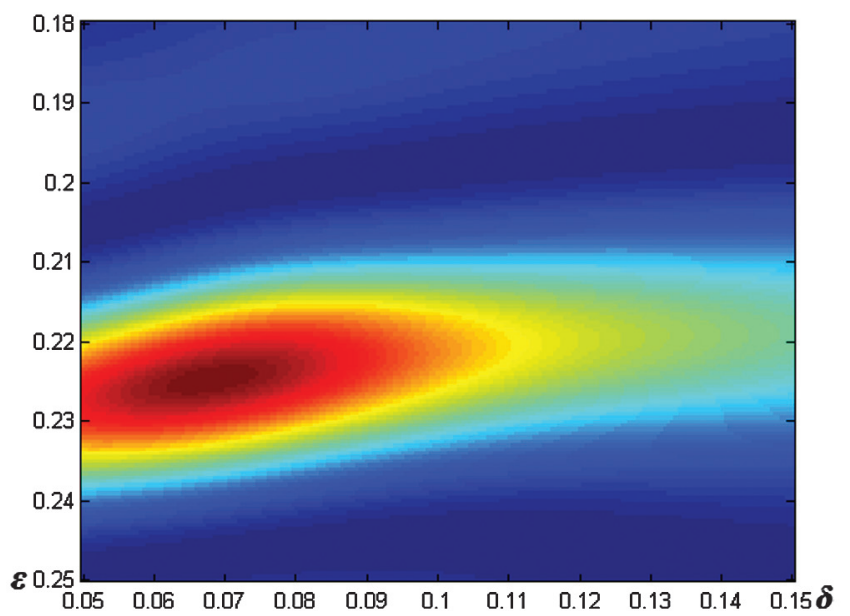

c)

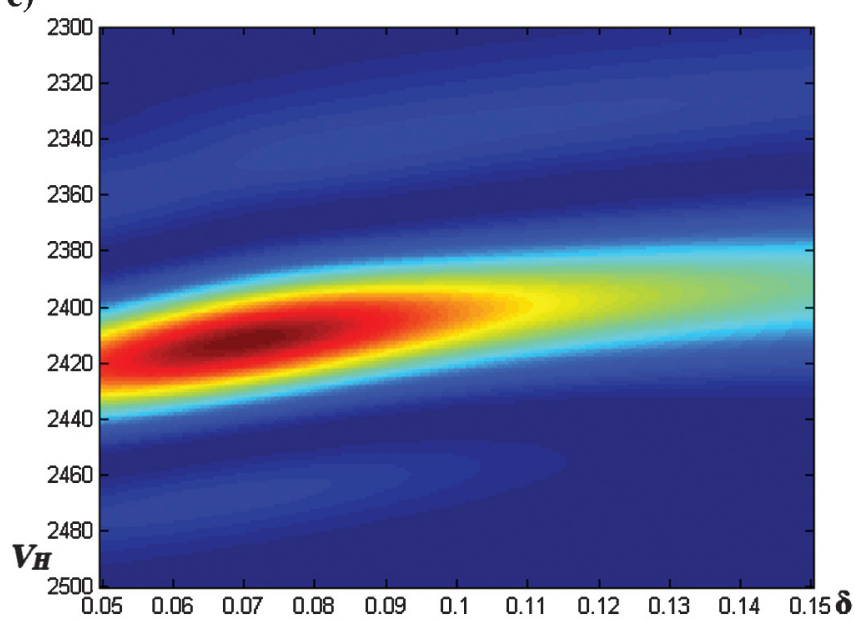

d)

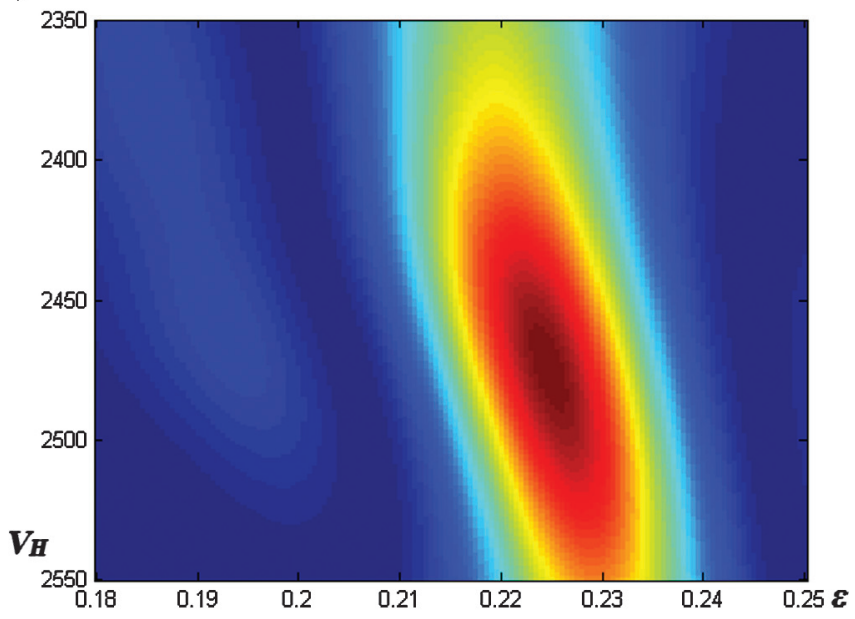

Figure 12. The semblance plots for different parameterizations computed for factorized model with $G_{2}=2 \mathrm{~s}^{-1}$ (a) $V_{N}$-fixed, $\varepsilon, \eta$; (b) $V_{N^{-}}$ fixed, $\varepsilon, \delta$; (c) $V_{0}$-fixed, $\delta, V_{H}$; and (d) $V_{N}$-fixed, $\varepsilon, V_{H}$. The anisotropy parameters can be evaluated from the coordinates of the maximal value of the semblance plot.

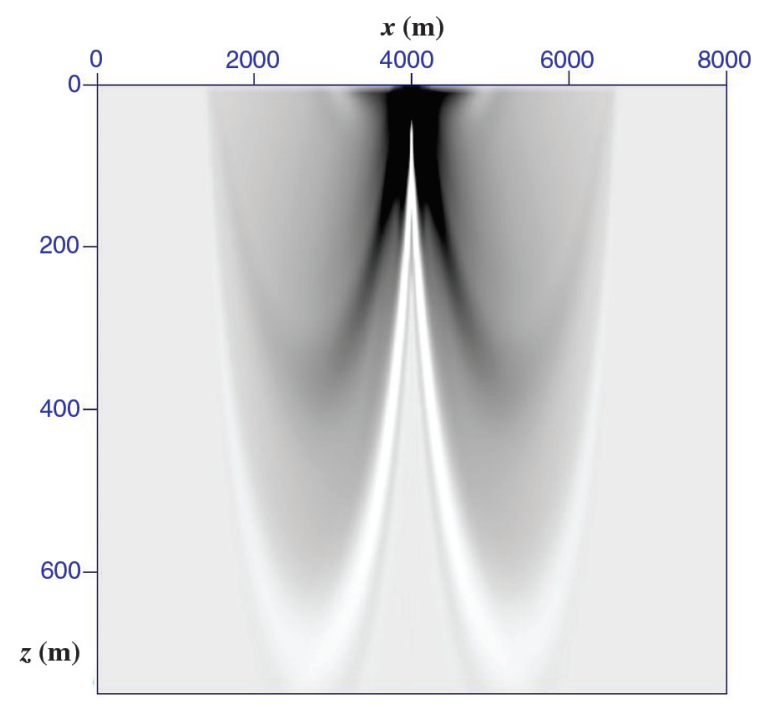

Figure 13. The ray trajectory of the diving waves. 

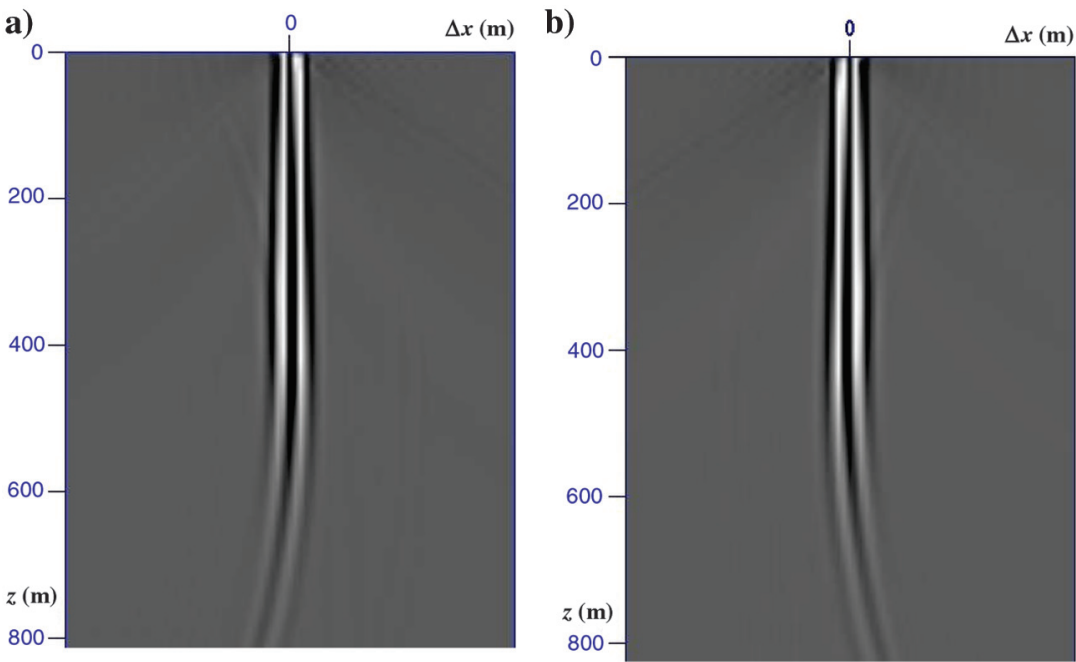

Figure 14. Subsurface image gathers in offset pulled from (a) $4400 \mathrm{~m}$ and (b) $4600 \mathrm{~m}$ of the model migrated with the correct velocity.

The semblance analysis exercise was mainly meant to analyze the role of parameterization in reducing the trade-off, but it also provides a valid tool for extracting effective anisotropic parameters corresponding to the depth covered by the diving waves. These effective values can serve as potential initial values for tomographic inversion or FWI.

\section{CONCLUSION}

We develop a method to estimate the anisotropy parameters from the residual moveout of diving waves in a factorized velocity model. We analyze different approximations for the imaging moveout and find that the second-order rational approximation $R_{2}$ is the most accurate one. By testing it with different anisotropy parameterizations, we select $\left(V_{N}, \varepsilon, \eta\right),\left(V_{N}, \varepsilon, \delta\right),\left(V_{0}, \varepsilon, V_{H}\right)$, and $\left(V_{N}, \varepsilon, V_{H}\right)$ as potentially the best combinations for this problem as they provide the best results even for large values of velocity gradients. We estimate the anisotropy parameters from the semblance analysis on residual moveout in the RTM image gathers. From the semblance plots, we observe that an increase in velocity gradient results in a decrease in the trade-off between anisotropy parameters. However, for the larger gradient the accuracy of our moveout equation reduces. Nevertheless, the anisotropy estimation using semblance analysis for all parameterizations is reasonably accurate even for large values of velocity gradients.

\section{ACKNOWLEDGMENTS}

We would like to thank W. Weibull for providing the modeling and RTM codes and C. Wang from Tongji University for his useful discussions. We acknowledge the China Scholarship Council and the ROSE Project for financial support and KAUST for its support. We also would like to acknowledge the associate editor and the reviewers for their instructive comments.

\section{APPENDIX A}

TAYLOR SERIES FOR RESIDUAL IMAGING MOVEOUT

To simplify the expression, we substitute the horizontal slowness by the following variable $s$, using

$$
p=\frac{1-s}{V_{0} \sqrt{1+2 \varepsilon}} .
$$

Then, the parametric expressions of $z_{p}(s)$ and $\Delta x_{p}(s)$ can be expanded in a series

$$
\begin{aligned}
z_{p}(s)= & n_{1} \sqrt{s}+n_{2} s+n_{3} s^{3 / 2}+n_{4} s^{2} \\
& +\cdots, \\
\Delta x_{p}(s)= & m_{1} \sqrt{s}+m_{2} s+m_{3} s^{3 / 2}+m_{4} s^{2} \\
& +\cdots,
\end{aligned}
$$

where $n_{j}, m_{j}, j=1, \ldots, 4$ are the series coefficients.

From series A-2, we define the series for $\Delta x_{p}\left(z_{p}\right)$ in the form

$$
\Delta x_{p}\left(z_{p}\right)=a_{1} z_{p}+a_{2} z_{p}^{2}+a_{3} z_{p}^{3}+a_{4} z_{p}^{4}
$$

The coefficients in series A-3 are given in terms of coefficients in series A-2 and A-3 as follows:

$a_{1}=\frac{m_{1}}{n_{1}}, \quad a_{2}=\frac{m_{2} n_{1}-m_{1} n_{2}}{n_{1}^{3}}$,

$a_{3}=\frac{m_{3} n_{1}^{2}+2 m_{1} n_{2}^{2}-m_{1} n_{1} n_{3}-2 n_{1} n_{2} m_{2}}{n_{1}^{5}}$,

$a_{4}=\frac{m_{4} n_{1}^{3}-3 m_{3} n_{1}^{2} n_{2}+5 m_{2} n_{1} n_{2}^{2}-5 m_{1} n_{2}^{3}-2 m_{2} n_{1}^{2} n_{3}+5 m_{1} n_{1} n_{2} n_{3}-m_{1} n_{1}^{2} n_{4}}{n_{1}^{7}}$

Finally, these coefficients take the form

$$
\begin{aligned}
& a_{1}=\sqrt{2} \sqrt{\varepsilon}, \quad a_{2}=-\frac{(1+2 \varepsilon) G}{2 \sqrt{2} \sqrt{\varepsilon} V_{0}} \\
& a_{3}=\frac{(1+2 \varepsilon)(-\eta+\varepsilon(3+4 \eta)) G^{2}}{6 \sqrt{2} \varepsilon^{3 / 2} V_{0}^{2}(1+2 \eta)}, \\
& a_{4}=\frac{(1+2 \varepsilon)\left(1-2 \eta-16 \varepsilon^{2}(1+\eta)+\varepsilon(2+4 \eta)\right) G^{3}}{32 \sqrt{2} \varepsilon^{5 / 2} V_{0}^{3}(1+2 \eta)} .
\end{aligned}
$$

Note that the lower order coefficients $a_{1}$ and $a_{2}$ do not depend on the anisotropy parameter $\eta$. 


\section{APPENDIX B}

\section{RATIONAL APPROXIMATION}

We define two types of rational approximations with the following forms:

$$
R_{1}=\frac{p_{1} z_{p}+p_{2} z_{p}^{2}}{1+q_{1} z_{p}}, \quad R_{2}=\frac{P_{1} z_{p}+P_{2} z_{p}^{2}+P_{3} z_{p}^{3}}{1+Q_{1} z_{p}+Q_{2} z_{p}^{2}}
$$

where $p_{1}, p_{2}, q_{1}, P_{1}, P_{2}, P_{3}, Q_{1}$, and $Q_{2}$ are the series coefficients. To compute these coefficients, we define the infinite depth limit

$$
a_{\infty}=\lim _{z_{p} \rightarrow \infty}\left(\frac{\Delta x_{p}}{z_{p}}\right) \text {. }
$$

The coefficients in the rational approximation $R_{1}$ can be represented by

$$
p_{1}=a_{1}, \quad p_{2}=\frac{a_{2} a_{\infty}}{a_{\infty}-a_{1}}, \quad q_{1}=\frac{a_{2}}{a_{\infty}-a_{1}},
$$

where the coefficients $a_{j}$ are given in equation A-5.

The coefficients in rational approximation $R_{2}$ can be given by

$P_{1}=a_{1}$

$P_{2}=\frac{a_{2}^{3}-2 a_{1} a_{2} a_{3}+a_{1}^{2} a_{4}+a_{2} a_{3} a_{\infty}-a_{1} a_{4} a_{\infty}}{a_{2}^{2}-a_{1} a_{3}+a_{3} a_{\infty}}$,

$P_{3}=\frac{\left(a_{3}^{2}-a_{2} a_{4}\right) a_{\infty}}{a_{2}^{2}-a_{1} a_{3}+a_{3} a_{\infty}}, \quad Q_{1}=\frac{a_{1} a_{4}-a_{2} a_{3}-a_{4} a_{\infty}}{a_{2}^{2}-a_{1} a_{3}+a_{3} a_{\infty}}$

$Q_{2}=\frac{a_{2} a_{4}-a_{3}^{2}}{a_{1} a_{3}-a_{2}^{2}-a_{3} a_{\infty}}$,

where the coefficients $a_{j}$ are given in equation A-5.

\section{APPENDIX C}

\section{DIFFERENT PARAMETERIZATIONS}

To analyze the impact of the different parameterizations on our analysis, we use three types of parameterizations: one velocity plus two anisotropy parameters, two velocities plus one anisotropy parameter, and three velocities. Different anisotropy parameters can be transferred by the following relations:

$$
\begin{aligned}
\eta & =\frac{\varepsilon-\delta}{1+2 \delta}, \quad V_{N}=V_{0} \sqrt{1+2 \delta}, \\
V_{H} & =V_{0} \sqrt{1+2 \delta} \sqrt{1+2 \eta},
\end{aligned}
$$

where $V_{0}$ is the P-wave vertical velocity on the surface, $V_{N}$ is the NMO velocity on the surface, and $V_{H}$ is the horizontal velocity on the surface. The value of the anisotropy parameters are set to $V_{0}=2 \mathrm{~km} / \mathrm{s}, G_{1}=1.5 \mathrm{~s}^{-1}, \varepsilon=0.22$, and $\eta=0.1$.

\section{Type one: One velocity + two anisotropy parameters}

The parameterizations given by type one are $\left(V_{0}, \varepsilon, \eta\right),\left(V_{0}, \delta, \eta\right)$, $\left(V_{0}, \delta, \varepsilon\right),\left(V_{N}, \varepsilon, \eta\right),\left(V_{N}, \delta, \eta\right),\left(V_{N}, \delta, \varepsilon\right),\left(V_{H}, \varepsilon, \eta\right),\left(V_{H}, \delta, \eta\right)$, and $\left(V_{H}, \delta, \varepsilon\right)$. We fix the first value of the velocity and evaluate the other two-anisotropy parameters through semblance analysis. Then, we convert the estimation errors into the same form $\Delta \tilde{\varepsilon}$ and $\Delta \tilde{\eta}$ to analyze the accuracy of different parameterizations by the relation $\Delta \tilde{\varepsilon} \approx \Delta \tilde{\delta}+\Delta \tilde{\eta}$.

\section{Type two: Two velocities + one anisotropy parameter}

The parameterizations in type two are $\left(V_{0}, \varepsilon, V_{N}\right),\left(V_{0}, \eta, V_{N}\right)$, $\left(V_{0}, \delta, V_{H}\right),\left(V_{0}, \eta, V_{H}\right),\left(V_{N}, \delta, V_{H}\right),\left(V_{N}, \varepsilon, V_{H}\right), \quad\left(V_{H}, \delta, V_{N}\right)$, and $\left(V_{H}, \varepsilon, V_{N}\right)$. We fix the first parameter of the velocity and evaluate the velocity and the anisotropy parameter through semblance analysis. Then, we convert the estimation errors into the same form $\Delta \tilde{\varepsilon}$ and $\Delta \tilde{\eta}$ to analyze the accuracy of different parameterizations using the following relations:

$\tilde{\delta}=\frac{\left(\frac{\tilde{V}_{N}}{V_{0}}\right)^{2}-1}{2}, \quad \tilde{\varepsilon}=\frac{\left(\frac{\tilde{V}_{H}}{V_{0}}\right)^{2}-1}{2}$,

$\tilde{\eta}=\frac{\left(\frac{\tilde{V}_{H}}{V_{N}}\right)^{2}-1}{2}=\frac{\left(\frac{V_{H}}{\tilde{V}_{N}}\right)^{2}-1}{2}, \quad \Delta \tilde{\varepsilon} \approx \Delta \tilde{\delta}+\Delta \tilde{\eta}$.

\section{Type three: Three velocities}

The parameterization in type three is $\left(V_{0}, V_{N}, V_{H}\right)$. We fix the first value of the velocity and evaluate the other two velocities through semblance analysis. Then, we again convert the estimation errors into the same form $\Delta \tilde{\varepsilon}$ and $\Delta \tilde{\eta}$ to analyze the accuracy of different parameterizations by the relations shown in equation $\mathrm{C}-2$.

The errors in anisotropy estimations $\Delta \tilde{\varepsilon}$ and $\Delta \tilde{\eta}$ for 18 parameterizations are shown in Table 1. From this table, one can see that the estimations from all parameterizations are reasonably accurate and the differences between them are very small.

\section{REFERENCES}

Alkhalifah, T., 1995, Efficient synthetic-seismogram generation in transversely isotropic, inhomogeneous media: Geophysics, 60, 1139-1150, doi: 10.1190/1.1443842.

Alkhalifah, T., 1998, Acoustic approximations for processing in transversely isotropic media: Geophysics, 63, 623-631, doi: 10.1190/1.1444361.

Alkhalifah, T., and R.-E. Plessix, 2014, A recipe for practical full-waveform inversion in anisotropic media: An analytical parameter resolution study: Geophysics, 79, no. 3, R91-R101, doi: doi: 10.1190/geo2013-0366.1.

Baker, G. A., J. Gammel, and J. G. Wills, 1961, An investigation of the applicability of the Padé approximant method: Journal of Mathematical Analysis and Applications, 2, 405-418, doi: 10.1016/0022-247X(61) 90019-1.

Kazei, V., V. Troyan, B. Kashtan, and W. Mulder, 2013, On the role of reflections, refractions and diving waves in full-waveform inversion: Geophysical Prospecting, 61, 1252-1263, doi: 10.1111/gpr.2013.61.issue-6.

Levin, F. K., 1996, Anatomy of diving waves: Geophysics, 61, 1417-1424, doi: 10.1190/1.1444066.

Rogister, Y., and M. A. Slawinski, 2005, Analytic solution of ray-tracing equations for a linearly inhomogeneous and elliptically anisotropic velocity model: Geophysics, 70, no. 5, D37-D41, doi: 10.1190/1.2049347. 
Sarkar, D., and I. Tsvankin, 2003, Analysis of image gathers in factorized VTI media: Geophysics, 68, 2016-2025, doi: 10.1190/1.1635055.

Shen, P., 2013, Subsurface focusing measurement of diving waves and its application to reflection tomography: 75th Annual International Conference and Exhibition, EAGE, Extended Abstracts, Th1005.

Sirgue, L., and R. G. Pratt, 2004, Efficient waveform inversion and imaging: A strategy for selecting temporal frequencies: Geophysics, 69, 231-248, doi: $10.1190 / 1.1649391$

Stovas, A., 2010, Kinematical characteristics of the factorized velocity model: Geophysical Prospecting, 58, 219-227, doi: 10.1111/gpr.2010 .58 .issue-2.
Stovas, A., and T. Alkhalifah, 2014, Analytical approximations of divingwave imaging in constant-gradient medium: Geophysics, 79, no. 4, S131S140, doi: 10.1190/geo2013-0386.1.

Tang, Y., S. Lee, A. Baumstein, and D. L. Hinkley, 2013, Tomographically enhanced full wavefield inversion: 83rd Annual International Meeting, SEG, Expanded Abstracts, 1037-1041.

Thomsen, L., 1986, Weak elastic anisotropy: Geophysics, 51, 1954-1966, doi: $10.1190 / 1.1442051$.

Virieux, J., and S. Operto, 2009, An overview of full-waveform inversion in exploration geophysics: Geophysics, 74, no. 6, WCC1-WCC26, doi: 10 $.1190 / 1.3238367$ 